\title{
Thromboinflammatory mechanisms in sickle cell disease - challenging the hemostatic balance
}

Haematologica 2020

Volume 105(10):2380-2390

\section{Correspondence:}

NICOLA CONRAN

conran@unicamp.br

ERICH V. DE PAULA

erich@unicamp.br

Received: March 5, 2020.

Accepted: April 29, 2020.

Pre-published: May 21, 2020.

doi:10.3324/haematol.2019.239343

(C)2020 Ferrata Storti Foundation

Material published in Haematologica is covered by copyright. All rights are reserved to the Ferrata Storti Foundation. Use of published material is allowed under the following terms and conditions:

https://creativecommons.org/licenses/by-nc/4.0/legalcode. Copies of published material are allowed for personal or internal use. Sharing published material for non-commercial purposes is subject to the following conditions:

https://creativecommons.org/licenses/by-nc/4.0/legalcode, sect. 3. Reproducing and sharing published material for commercial purposes is not allowed without permission in writing from the publisher.
Nicola Conran and Erich V. De Paula

Hematology Center, University of Campinas, UNICAMP, Cidade Universitária, Campinas-SP, Brazil.

\section{ABSTRACT}

ickle cell disease (SCD) is an inherited hemoglobinopathy that is caused by the presence of abnormal hemoglobin $\mathrm{S}(\mathrm{HbS})$ in red blood cells, leading to alterations in red cell properties and shape, as the result of $\mathrm{HbS}$ dexoygenation and subsequent polymerization. The pathophysiology of SCD is characterized by chronic inflammatory processes, triggered by hemolytic and vaso-occlusive events, which lead to the varied complications, organ damage and elevated mortality seen in individuals with the disease. In association with activation of the endothelium and leukocytes, hemostatic alterations and thrombotic events are welldocumented in SCD. Here, we discuss the role of inflammatory pathways in modulating coagulation and inducing platelet activation in SCD, due to tissue factor activation, adhesion molecule expression, inflammatory mediator production and the induction of innate immune responses, among other mechanisms. Thromboinflammatory pathways may play a significant role in some of the major complications of SCD, such as stroke, venous thromboembolism and possibly acute chest syndrome, besides exacerbating the chronic inflammation and cellular interactions that trigger vaso-occlusion, ischemia-reperfusion processes, and eventually organ damage.

\section{Introduction}

The sickle cell disorders are inherited hemoglobinopathies that occur with an estimated incidence of between 300,000 to 400,000 births every year worldwide. ${ }^{1}$ These disorders are caused by mutations in the $H B B$ gene (encoding hemoglobin subunit $\beta$ ), resulting in an amino-acid substitution in the $\beta$-globin chain. Homozygosity of the mutated sickle hemoglobin gene incurs sickle cell anemia, while compound heterozygosity with another $H B B$ gene mutation causes variations of sickle cell disease (SCD). ${ }^{2}$ The altered hemoglobin $\mathrm{S}(\mathrm{HbS})$ that is produced polymerizes in the red blood cell (RBC) when deoxygenated, provoking the complex pathophysiology of the disease. SCD, particularly sickle cell anemia, is characterized by a high burden of morbidity and reduced life expectancy, ${ }_{1}^{1}$ due to hemolytic anemia and vaso-occlusive processes, which result in the painful vasoocclusive episodes that can lead to the hospitalization of patients and contribute to chronic organ damage. Other complications of SCD vary widely from patient to patient, but include both acute and chronic complaints such as stroke, acute chest syndrome, retinopathy, renal disease, osteonecrosis, cardiovascular complications and leg ulcers. ${ }^{1}$

\section{Overview of the pathophysiology and inflammatory mechanisms of sickle cell disease}

$\mathrm{HbS}$ polymerization induces RBC sickling and impairs cell deformability, altering blood rheology and the RBC membrane. Sickle RBC are dense, and tend to be more dehydrated and more adhesive to the endothelium; they also generate reactive oxygen species, release more pro-inflammatory microparticles containing heme 
and can activate the complement and coagulation systems. ${ }^{3}$ These fragile and rigid RBC lyse easily in the circulation, both extravascularly and intravascularly, and have a significantly shorter lifespan than that of normal RBC. ${ }^{2}$ The ensuing release of non-compartmentalized (or cellfree) hemoglobin from ruptured RBC has significant pathophysiological consequences in SCD, in which endogenous scavenging mechanisms are overwhelmed. ${ }^{4}$ Cell-free hemoglobin reacts rapidly with vascular nitric oxide (NO), disrupting vasodilation and favoring cellular activation. Furthermore, oxidative reactions with cell-free hemoglobin lead to the release of heme and free iron. ${ }^{5}$ Similarly, the ischemia-reperfusion events that result from vaso-occlusive processes in the microcirculation also activate inflammatory cells and initiate sterile inflammatory processes and oxidant generation. ${ }^{6}$

Endothelial activation, a hallmark of SCD pathophysiology, augments pro-inflammatory molecule generation, and in vitro and in vivo studies suggest that it is the adhesion and capture of activated and more adhesive red cells, leukocytes and platelets to the endothelium of the blood vessel wall that trigger vaso-occlusion. ${ }^{6-8}$ Endothelial cells and leukocytes also play major roles in generating the plethora of pro-inflammatory molecules that are upregulated in SCD, including cytokines (such as tumor necrosis factor and interleukin-1 $\beta$ ), chemokines, growth factors, eicosanoids and peptides, all of which can further stimulate cells, and induce expression of surface adhesion molecules.9 Activation of platelets is another characteristic of SCD, in which these cells participate in the generation of inflammatory molecules and heterocellular interactions that propagate vaso-occlusive processes, as will be discussed later. ${ }^{10,11}$ Additionally, functional asplenia renders individuals with SCD (particularly children) more susceptible to bacterial infections, ${ }^{12}$ and gut permeability is reportedly augmented in individuals, ${ }^{13,14}$ leading to a further exacerbation of inflammatory processes, mediated by innate immune mechanisms generated in response to exposure to pathogens and gut microbiota-derived molecules. ${ }^{13}$ The inflammatory mechanisms of SCD have been comprehensively reviewed recently, ${ }^{9,15}$ with evidence from ex vivo and in vivo studies illustrating the complex pathophysiology of SCD which involves a vicious circle of $\mathrm{RBC}$ alterations, hemolytic events, vaso-occlusive processes and a chronic inflammatory state involving several molecular pathways.

\section{Sickle cell disease as a prothrombotic state}

The characterization of SCD as a prothrombotic state is supported by extensive laboratory and clinical data. From a laboratory perspective, almost every compartment of hemostasis has been evaluated in patients with SCD, mainly at steady-state but also during vaso-occlusive crises, in several independent cohorts. ${ }^{16}$ Although there are some inconsistencies between results, possibly reflecting differences in study design (e.g. issues related to sample collection, processing and analysis) and patients' heterogeneity (e.g. definition of vaso-occlusive crises), one can safely state that SCD is a condition in which the hemostatic balance is clearly tipped towards a prothrombotic state. Details about these mechanisms have been the subject of excellent recent reviews, ${ }^{17-19}$ so that in an attempt to convey a broader view of these studies, we will list this evi- dence according to whether studies were mainly descriptive, or whether they also provided mechanistic insights regarding this hypercoagulable state.

Descriptive studies comprise those that measured markers that are normally changed in response to the activation of hemostasis, and most likely represent consequences thereof, such as studies showing that SCD patients have increased levels of thrombin-antithrombin complexes, Ddimer, prothrombin fragment 1.2, factor (F) VIII and fibrinogen. ${ }^{20-22}$ This group includes studies that showed that SCD is also associated with hypercoagulable profiles in global assays of hemostasis, such as the thrombin generation assay and thromboelastometry, ${ }^{23}$ which are relevant because they provide information on the net effect of the complex hemostatic abnormalities found in patients. The second group comprises studies that evaluated parameters which, in addition to illustrating that hemostasis is activated, could also represent mechanisms by which the prothrombotic state is either initiated and/or perpetuated. These include studies showing decreased levels of natural anticoagulants such as protein $C$ and protein $S,{ }^{17,20}$ increased expression and/or activity of tissue factor (TF) in whole blood, monocytes and circulating endothelial cells, ${ }^{24-26}$ increased levels of von Willebrand factor (vWF) coupled with decreased levels of ADAMTS-13, ${ }^{27}$ increased numbers of TF- and phosphatidylserine-bearing microparticles, $^{21,22,28}$ decreased levels of contact pathway factors (FXII, prekallikrein and high molecular weight kininogen), ${ }^{19}$ and increased markers of neutrophil extracellular trap (NET) formation. ${ }^{29}$ A non-exhaustive summary of these abnormalities is presented in Table 1.

Importantly, most of these phenomena have also been

Table 1. Key hemostatic abnormalities in clinical studies of sickle cell disease.

Biomarkers of increased activation of hemostasis

$\uparrow$ Thrombin antithrombin complexes

$\uparrow$ Prothrombin fragment 1.2

$\uparrow$ D-dimer

$\uparrow$ Direct markers of thrombin generation (thrombin generation assay)

Hypercoagulable pattern in viscoelastic assays

(thromboelastometry assay)

$\uparrow$ Factor VIII

$\uparrow$ Fibrinogen

$\uparrow$ Platelet $\alpha_{i \mathrm{ib}} \beta_{3}$ integrin activation

$\uparrow \mathrm{CD} 40 \mathrm{~L}$

Likely involved in the initiation and/or perpetuation

of hemostasis activation

$\uparrow$ Tissue factor expression and/or activation

$\downarrow$ Natural anticoagulants (proteins $\mathrm{C}$ and $\mathrm{S}$ )

$\uparrow$ Von Willebrand factor reactivity with relatively low ADAMTS13

$\uparrow$ Phosphatidylserine-bearing red blood cell microparticles

$\uparrow$ Plasminogen activator inhibitor-1

$\uparrow$ Markers of neutrophil extracellular trap formation

$\downarrow$ Contact pathway factors (factor XII, prekallikrein and high molecular weight kininogen)

$\uparrow$ Platelet counts

$\uparrow$ P-selectin expression 
observed in the two most representative animal models of SCD, which have been critical to our understanding of the mechanisms of hypercoagulability in this condition. First, it was shown that ischemia-reperfusion injury, one of the key pathogenic elements of SCD, triggers endothelial TF expression ${ }^{30}$ which was later shown to be involved mostly in the inflammatory spectrum of the pathogenesis of SCD, as we shall discuss later. The role of TF was demonstrated by a study in which inhibition of TF with anti-TF antibodies reduced both coagulation activation and inflammation, but that the former effect was not dependent on endothelial TF. ${ }^{31}$ The role of neutrophils in the formation of microvascular thrombi was also confirmed in SCD animal models, ${ }^{8,32}$ while further in vivo studies established that excess heme activates TF-mediated coagulation, and also confirmed the significance of leukocyte TF in this effect. ${ }^{33}$ The relevance of coagulation activation for the pathogenesis of SCD was nicely illustrated by demonstrations that downregulating thrombin generation by either genetic ${ }^{34}$ or pharmacological ${ }^{35}$ approaches reduced inflammation and improved clinical manifestations in SCD mice. The participation of fibrinogen in this cycle as more than a biomarker was also demonstrated by studies using SCD mice. ${ }^{36}$ Additionally, observations of clot formation in SCD mice, and in human SCD blood samples, show that the interaction of RBC with each other and with other cells inside venous thrombi may make an additional contribution to hypercoagulability in SCD ${ }^{37}$ Finally, dense sickle cells and hemolysis are implicated in activation of the complement system, an innate immune defense cascade, in patients with $S C D,{ }^{38}$ and complement activation by hemolytic and ischemia-reperfusion mechanisms are reported to promote vaso-occlusive processes and prothrombotic responses in mice with SCD. ${ }^{39}$

From a clinical perspective, the long-described association of SCD with ischemic stroke, and the more recently characterized risk of silent cerebral infarcts and venous thromboembolism (VTE) provide definite evidence that SCD represents a prothrombotic state. Data from the Cooperative Study on Sickle Cell Disease revealed an incidence of $0.61 / 100$ patient-years for stroke, ${ }^{40}$ and of 7.6/1,000 patient-years for VTE ${ }^{41}$ in patients with the most severe forms of SCD, yielding cumulative rates as high as $24 \%$ and $11.3 \%$ by the age of 40 and 45 years for stroke and VTE, respectively. For reasons that are yet to be determined, but that indicate a somewhat different pathogenesis compared to that of other conditions associated with VTE, only pulmonary embolism, but not deep vein thrombosis, is more frequent in SCD. ${ }^{41}$ Again, excellent reviews have been recently published on these manifestations and their relationship with coagulation abnormalities. ${ }^{42}$ Collectively, laboratory data encompassing almost every known biomarker of hemostasis, coupled with clinical and epidemiological findings provide robust evidence of the presence of a prothrombotic state in SCD.

Although stroke and VTE represent the most straightforward thrombotic manifestations in SCD, other clinical manifestations of SCD, whose pathogenesis involves vaso-occlusive processes in the microcirculation. are normally associated with the prothrombotic phenotype described in these patients. ${ }^{18,19,43}$ These include acute chest syndrome, acute painful episodes, pulmonary hypertension and even chronic organ failure attributed to chronic ischemia. In fact, despite the intuitive association that can be established between these manifestations and a pro- thrombotic hemostatic balance, as well as the demonstration that downregulation of thrombin generation can improve these manifestations in mouse models of SCD, 34,35 their association with classical markers of hemostasis activation in patients with SCD is not consistent, ${ }^{44.47}$ in what remains one of the most intriguing issues in this area of research. As we shall discuss in the next session, the application of the well-established concept that hemostasis activation cannot be dissociated from the immune response in the field of $\mathrm{SCD}^{15,18,43}$ has been instrumental to better understanding this issue, paving the way for novel therapeutic advances.

\section{Thromboinflammation and sickle cell disease}

\section{Hemostasis as part of the immune response}

Hemostasis and innate immunity are both involved in the immediate response to external and internal agents that threaten tissue/organ integrity, such as pathogens and trauma. Classically viewed as two independent systems, initial discussions on their inter-relationship derived mostly by teleological reasoning based on the example of the horseshoe crab, an extant organism with 450 million years of phylogenetic history in which breeches in tegument and invasion by pathogens are tackled by a single cellbased system that senses endotoxins and triggers a cascade of events that closely resembles the architecture of the hemostatic system. ${ }^{48}$ However, in the last two decades several lines of empirical evidence have emerged to confirm the intimate crosstalk between hemostasis and innate immune responses, and the terms thromboinflammation and immunothrombosis ${ }^{49,50}$ have been introduced to summarize the concept that the cellular programs driving the formation of microthrombi in a spatially- and temporallyorganized fashion can be elicited in response to pathogens and/or tissue damage, in order to facilitate pathogen removal and tissue repair.

Evidence supporting the association between hemostasis and innate immunity include: (i) studies in animal models of sepsis and/or infection in which discrete elements of hemostasis are reportedly up- or down-regulated; despite some heterogeneity, a less effective hemostatic system is generally associated with increased pathogen multiplication and, in some cases, poorer outcomes (recently reviewed elsewher $\left.{ }^{51}\right)$; (ii) demonstrations that critical elements of hemostasis such as TF, thrombin and activated protein $\mathrm{C}$ also signal through pathways that regulate the immune response, independently of their role in fibrin formation; ${ }^{52}$ (iii) examples of microorganisms whose virulence factors are proteins that disrupt local hemostatic balance, such as Yersinia pestis, ${ }^{53}$ (iv) findings that byproducts of coagulation activation, such as peptides derived from thrombin and fibrinogen, have antibacterial properties; $;^{51}$ (v) intravital imaging studies showing that leukocytes are not only found in arterial and venous thrombi, but that localized coagulation activation and fibrin formation also seem to direct their adhesion to the endothelium and migration into tissues; $;^{54}$ and more recently, (vi) the demonstration that downregulation of critical elements of the innate immune response can protect mice from experimental thrombosis, with the example of NET formation being most paradigmatic..$^{55}$ Though less straightforward, studies using system biology approaches, capable of analyzing the complex interplay between several systems, 
have also provided indirect evidence that pathways involved in tissue repair, such as hemostasis, angiogenesis and complement, are associated with innate immune responses in inflammatory conditions such as sepsis and SCD. ${ }^{56}$ Taken together, these data support the concept that, in a disease long-recognized as a systemic inflammatory condition, such as SCD, the sustained activation of immunothrombosis underlies the consistent, yet heterogeneous, increase in biomarkers of coagulation activation that is observed in these patients.

\section{Activation of hemostasis in inflammatory states and in sickle cell disease}

Understanding how hemostasis is activated as part of inflammatory responses is critical to our understanding of the pathogenesis of hypercoagulability in SCD, since the regulation of these mechanisms may be somewhat different from the classical pathways of hemostasis activation in non-inflammatory states, which consist mostly of $\alpha_{\text {iib }} \beta_{3}$ integrin-mediated platelet activation, and of the contact and activation of TF with activated FVII on cellular surfaces. ${ }^{52}$ According to recent reports, these elements can be regulated and activated by different pathways during inflammation, and new players may contribute to the activation of hemostasis.

As previously mentioned, thrombin and activated protein $\mathrm{C}$ can also activate pro-inflammatory and pro-angiogenic pathways by signaling through protease-activated receptors (PAR). ${ }^{57}$ Of note, this pathway is thought to require much lower concentrations of thrombin than those required for overt conversion of fibrinogen into fibrin, ${ }^{58}$ consistent with a role for localized thrombin generation in the regulation of the immune response. In the context of SCD, the relevance of coagulation-independent functions of these proteins has been demonstrated in animal models in which low TF levels ( 1\%) in nonhematopoietic cells attenuated inflammation, but not activation of coagulation. ${ }^{31}$ Similarly, PAR-2 deficiency has been shown to attenuate lung inflammation and interleukin-6 production, without affecting classical markers of coagulation activation. ${ }^{35}$ More recently, PAR-1 deficiency or PAR-1 inhibition was found to decrease heme and lipopolysaccharide-induced vaso-occlusion. ${ }^{59}$

Another mechanism of coagulation activation and amplification by inflammation is mediated by components of NET (DNA and histones) released from neutrophils. ${ }^{60}$ The participation of NET in the pathogenesis of SCD was suggested by the association of increased biomarkers of NET formation with the severity of acute vasoocclusive crises in these patients, ${ }^{29}$ as well as by data from animal models of SCD. ${ }^{61}$ A third mechanism by which hemostasis can be activated during inflammatory states is through the intrinsic pathway involving FXII, prekallikrein, high molecular weight kininogen and FXI. Evidence that this pathway contributes to hypercoagulability led to a series of ongoing clinical trials with FXI inhibitors in patients with thrombosis. ${ }^{62}$ The role of the intrinsic pathway in coagulation activation in SCD has been demonstrated in animal models of FXII deficiency and consequently reduced thrombin generation. ${ }^{19}$ Interestingly, several molecules (e.g. DNA, polyphosphates), some of which are increased in SCD, have been shown to activate or amplify this pathway in vitro, ${ }^{63}$ although the relevant elements that regulate the intrinsic pathway in SCD patients remain to be determined.
More recently, two additional pathways have been added to the list of potential mediators of hemostasis activation during inflammation. The angiopoietin/Tie-2 axis has been shown to participate in the activation of hemostasis during sepsis, ${ }^{64}$ and the CLEC-2/podoplanin pathway, involved in securing hemostasis during inflammation, has also been associated with outcomes that can fit into the spectrum of immunothrombosis in both animal models ${ }^{65}$ and humans. ${ }^{66}$ Exploring their role in the context of SCD should be of interest. In fact, although it is tempting to speculate that mechanisms that drive hemostasis activation in models of inflammation, such as sepsis, might also be relevant to the pathogenesis of SCD, one should not ignore the recent evidence suggesting that, in addition to the inflammatory processes of ischemia /reperfusion that typify SCD, ${ }^{6}$ one very specific characteristic of SCD, namely sustained hemolysis, could well be central to the activation of thromboinflammatory pathways in this disease.

\section{Hemolysis as a driver of thromboinflammation}

Intravascular hemolysis is now widely accepted as a major pro-inflammatory mechanism that significantly influences the pathophysiology of numerous diseases and disorders, including SCD. ${ }^{4}$ As previously mentioned, the reaction of cell-free hemoglobin, released during hemolysis, with vascular $\mathrm{NO}$ incurs immediate effects on endothelial and vascular function. Oxidative reactions with hydrogen peroxide and lipid peroxide also yield the $\mathrm{Hb}-\mathrm{Fe}^{3+}$ product in the circulation and tissues, ${ }^{5}$ which readily releases its heme group, a hydrophobic molecule that has recently been designated as a major erythrocytic danger-associated molecular pattern (DAMP) that activates and amplifies inflammatory mechanisms. ${ }^{6}$ Evidence of the pro-inflammatory effects of heme continues to advance with both in vitro and in vivo studies demonstrating the role of this molecule in multiple inflammatory pathways, due to the induction of oxidant reactions, lipid peroxidation $^{5}$ and activation of receptors involved in innate immune responses. ${ }^{68}$ Notably, an intricate association exists between free heme and thromboinflammation, which promotes activation of hemostasis and its components.

Heme has a significant effect on the endothelium, which plays a primary role in hemostasis due to its expression of vWF, TF exposure and major thrombin generation, among other properties. ${ }^{69}$ Non-protein associated cell-free heme can directly activate the endothelium via binding to toll-like receptor (TLR)- $4^{68}$ or via the transfer of heme from heme- and hemoglobin-laden microparticles derived from erythrocytes. ${ }^{70}$ In addition to inducing oxidative stress, heme-mediated activation of the endothelium results in mobilization of vWF and P-selectin from the WeibelPalade bodies to the endothelial cell surface, as well as upregulation of other adhesion molecules, which may promote platelet and leukocyte recruitment to blood vessel walls. ${ }^{68,71}$ TLR-4-mediated activation of pro-inflammatory nuclear factor- $\kappa \mathrm{B}$ transcription factor activity ${ }^{68}$ may also upregulate endothelial cytokine generation, as well as TF expression. ${ }^{72}$ Furthermore, heme can induce reactive oxygen species-dependent NLRP3 inflammasome assembly in endothelial cells, with consequent processing of interleukin- $1 \beta,^{73}$ a potent pro-inflammatory cytokine that 
may amplify platelet activation and clot formation, among other effects. ${ }^{74}$ Importantly, heme exposure induces C3 deposits on glomerular endothelial cells, amplifying innate immune complement activation and local thrombotic lesions, ${ }^{75}$ while the stimulation of TLR-4-dependent endothelial P-selectin expression by experimental hemolysis has been reported to trigger complement activation via the non-covalent anchoring of C3 activation fragments in the murine liver, leading to liver damage. ${ }^{76}$

Platelet activation and aggregation are central to thrombus formation; importantly, the decrease in NO bioavailability that occurs during intravascular hemolysis, in addition to modulating vasodynamics, may repress the major inhibitory effect that NO has on the expression and activation of adhesion molecules on cell surfaces. In particular, $\mathrm{NO}$ inhibits the activities of the fibrinogen and collagen receptors $\left(\alpha_{\mathrm{ib}} \beta_{3} \text { and } \alpha_{2} \beta_{1} \text { integrins, respectively }\right)^{77,78}$ on the platelet surface and, therefore, the hemolysis-induced reduction in NO may play a role in thrombus formation. Hemolysis also activates platelets due to the release of intra-erythrocytic adenosine 5'-diphosphate (ADP), via activation of P2Y1, P2Y12, and P2X1 ADP receptors on the platelet surface. ${ }^{79}$ Additionally, heme directly mediates the activation and death of platelets via lipid peroxidation and subsequent ferroptosis, an iron-dependent form of nonapoptotic cell death, ${ }^{80}$ and oxidized heme induces divalent action-dependent platelet aggregation, associated with thromboxane formation and fibrinogen binding. ${ }^{81}$

Hemolysis and products of hemolysis have long been associated with modulation of coagulation. The administration of hematin to patients with porphyria reportedly activated coagulation parameters in some patients, prolonging prothrombin and partial thromboplastin times, as well as increasing fibrinogen-degradation products, among other effects, ${ }^{69,82}$ although in vitro studies have shown the inhibition of coagulant factors and clot lysis by hematin. ${ }^{83}$ In addition to its aforementioned stimulation of TF expression by endothelial cells, in vivo administration of heme also stimulates TF production in leukocytes ${ }^{33}$ and may activate coagulation, at high concentrations, via stimulation of TF and the extrinsic pathway. ${ }^{33,84}$ In contrast, free heme binds to several sites on FVIII with high affinity and at high concentrations can inhibit its activity in vitro; ${ }^{35}$ FVIII-heme interactions can, however, be inhibited by vWF, whose release from endothelial cells is also stimulated by heme. ${ }^{68}$ In turn, multimeric vWF can be bound by extracellular hemoglobin and bilirubin (a degradation product of heme), impairing its enzymatic cleavage by the ADAMTS-13 protein, resulting in increased prothombotic activity. With regards to final clot formation, hemolysis has been suggested to exacerbate hyperfibrinolysis and facilitate uncontrolled bleeding, with red cell lysate accentuating tissue plasminogen activator-mediated fibrinolysis ${ }^{86}$ In contrast, hematin and the products of heme degradation, iron, bilirubin and carbon monoxide, are known to interact with fibrinogen, and induce fibrin formation or decrease fibrinolysis, possibly accelerating the formation of resistant clots. ${ }^{33,87,88}$ As such, heme has a clear role in triggering the initiation of coagulation, although its effects on the propagation of this system are not as well understood. ${ }^{69}$

Cell-free heme and heme-loaded microvesicles also modulate the complement system ${ }^{89}$ which integrates closely with innate immunity and coagulation..$^{90}$ This mechanism is particularly relevant given that paroxysmal nocturnal hemoglobinuria, a disease characterized by complement- driven chronic hemolysis, predisposes patients to thrombosis ${ }^{90}$ among other complications. In particular, heme overactivates the alternative complement pathway by binding to the C3 molecule, and increasing its spontaneous hydrolysis; the anchoring of complement activation fragments to erythrocytes and endothelial cells may result in their destruction (and further hemolysis) or activation, respectively, ${ }^{69}$ and thromboinflammation. The classical complement pathway, in contrast, may be inhibited by heme due to heme binding to $\mathrm{C} 1 \mathrm{q}$ and reduced C3 convertase formation and $\mathrm{C} 3 \mathrm{~b}$ deposition. ${ }^{69}$ Importantly, anti-inflammatory intravenous immunoglobulin scavenges heme and can inhibit heme-mediated activation of the complement system on the surface of human endothelial cells. ${ }^{91}$

Endogenous systems are in place to ensure that cell-free hemoglobin and heme are inactivated and eventually degraded in the organism during hemolytic processes. Haptoglobin and hemopexin are plasma proteins with very high binding affinities for hemoglobin and heme, ${ }^{92}$ respectively, rendering them relatively non-reactive and delivering them to macrophages and hepatocytes, respectively, for endocytosis and degradation of their heme moieties by the heme oxygenase (HO-1) enzyme, which is upregulated in response to the presence of heme. ${ }^{9}$ The anti-inflammatory properties of HO-1 are well documented, and HO-1 activity in SCD mice appears to offer some protection from thrombosis. ${ }^{93}$ Furthermore, HO-1, and the products of heme degradation, were recently shown to decrease clot size in a murine venous thrombosis model, while clot size is augmented in hemopexin-knockout mice. ${ }^{94}$

The equilibrium between free and protein-associated heme is vital for determining the pro-inflammatory capacity of heme and it has been suggested that, in fact, it is unlikely that equilibrium conditions that lead to inflammation and, therefore, thromboinflammation could ever occur in vivo. ${ }^{92}$ However, in diseases characterized by severe intravascular hemolysis, such as SCD, plasma haptoglobin and hemopexin levels are often depleted, ${ }^{95,96}$ meaning that the free and bound heme equilibrium may be tipped, leading to the thromboinflammatory consequences of hemolysis.

\section{Ischemia-reperfusion injury and thromboinflammation}

Ischemia-reperfusion injury is a feature of multiple clinical disorders, including myocardial infarction and ischemic stroke. ${ }^{97}$ Processes of ischemia, followed by reperfusion, occur in SCD during vaso-occlusive episodes, although their preponderance in the microvasculature means that they generally have comparatively fewer acute consequences than those occurring in larger vessels; however, their recurrent nature means that these processes contribute significantly to local tissue and organ damage. The twophase character of ischemia-reperfusion injury results in the generation of danger signals, or DAMP, from damaged cells, ${ }^{9}$ and activation of components of the innate immune system, resulting both from the interruption of the tissue's vascular supply during vaso-occlusion and from the reoxygenation of the vasculature upon resumption of blood flow. ${ }^{98}$ The generation of reactive oxygen species is a particularly damaging feature of the reoxygenation stage..$^{98}$

As described, thromboinflammatory mechanisms are significant in triggering the cellular and molecular events 
that lead to vaso-occlusion, but ischemia and reperfusion events further exacerbate inflammatory responses including thromboinflammatory events, reflecting, once again, the cyclic nature of SCD pathophysiology. Further activation of platelet function, in association with altered endothelial hemostatic activity and TF-thrombin pathway activation are particular features of ischemiareperfusion. ${ }^{99,100}$ Mice subjected to arterial occlusion, followed by reperfusion, demonstrate platelet adhesion to the endothelium and the formation of aggregates, ${ }^{101}$ while depletion of platelets, or inactivation of their function, can significantly reduce ischemia-reperfusion in multiple organs. ${ }^{97}$ In turn, hypoxia-reoxygenation processes induce RBC sickling in mice with SCD, generating oxidative stress and the endothelial cell activation that contributes to thromoboinflammation and TF expression..$^{98}$ As such, hemostatic alterations and thrombotic processes appear to be intimately associated with inflammatory mechanisms in SCD that are triggered by hemolysis and ischemiareperfusion injury. Activation of endothelial cells and leukocytes is well-documented in SCD and plays a role in eliciting alterations in coagulation pathways and the activation of platelets, due to TF release, adhesion molecule expression, inflammatory mediator production and the induction of innate immune responses (Figure 1), contributing to further inflammation and tissue damage.

\section{Sickle cell disease thromboinflammation and platelet activation}

Beyond their role in thrombosis and hemostasis, platelets are increasingly recognized to be key regulators of inflammatory responses, in both sterile and infectious situations. ${ }^{102}$ The basis of platelet activation and abnormal platelet function in SCD is not clear; as mentioned, hemolysis may be a major factor in platelet activation due to ensuing $\mathrm{NO}$ depletion and the release of the platelet agonist, ADP, in addition to exposure of RBC phosphatidylserine and enhanced thrombin generation. 21,79 Platelets also express TLR4, which can be activated by pathogen-derived lipopolysaccharide and, likely, heme. ${ }^{103}$ Platelet activation in SCD is characterized by differentiated gene expression, ${ }^{104}$ augmented adhesion molecule activity, ${ }^{105,106}$ and the increased circulation of platelet microparticles and platelet-derived proteins. ${ }^{107,108}$ Persistent platelet activation, while apparently leading to some refractory inhibition of platelet aggregation, ${ }^{109}$ plays an increasingly recognized role in SCD pathophysiology and appears to be further exacerbated during acute vaso-occlusive episodes, ${ }^{110}$ while probably representing a key mediator of inflammatory pathways.

The increased expression and activity of adhesion molecules, such as $\alpha_{\mathrm{Ib}} \beta_{3}$, P-selectin, and glycoprotein Ib $\alpha$, on SCD platelets facilitate the cells' adhesion to the endothelium and induce endothelial activation ${ }^{11,105,111}$ and also permit the formation of P-selectin-mediated heterocellular aggregates between platelets and leukocytes and/or erythrocytic cells, which play an increasingly recognized role in the ini-

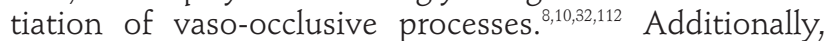
platelets are major sources of inflammatory mediators and contribute to chronic inflammation in SCD by releasing inflammatory cytokines, such as platelet factor 4, interleukin-1 $\beta$, LIGHT (TNFSF14), and CD40L; ${ }^{11,106,113}$ elevated levels of circulating CD40L have been associated with the occurrence of acute chest syndrome in SCD. Activated platelets are also a major source of thrombospondin-1, another protein that has been related to the incidence of acute chest syndrome and vaso-occlusive episodes ${ }^{108}$ and that, interestingly, triggers shedding of RBC microparticles, causing vaso-occlusion, in mice with SCD. ${ }^{114}$ Furthermore, the platelet NLRP3 inflammasome is reportedly upregulated in SCD and may, therefore, play a major role in the processing of interleukin-1 $\beta$ and interleukin-18, which have important pro-inflammatory roles in the disease. ${ }^{115}$

\section{Thrombotic manifestations of sickle cell disease in the spectrum of thromboinflammation}

Despite our current knowledge about the characteristics and mechanisms by which hemostasis is activated in SCD, the precise role of hemostasis in the pathogenesis of complications of the disease has not been completely clarified, as the association between classical hemostatic biomarkers and the occurrence of clinical manifestations of SCD is not straightforward, ${ }^{42}$ and results from clinical trials using antithrombotic agents have yielded heterogeneous results. On the other hand, significant clues about the relevance of hemostasis activation in SCD include data from animal models showing that reductions of prothrombin to levels normally observed in anticoagulated patients improved organ damage in SCD mice and even mortality, ${ }^{34}$ in association with attenuation of inflammation and vaso-occlusion after downregulation of PAR-1, PAR-2 and TF. ${ }^{35,59,116}$ Interestingly, in sickle cell disorders with genotypes that are generally regarded as being associated with a milder phenotype, such as $\mathrm{HbSC}$ disease and $\mathrm{S} / \boldsymbol{\beta}^{+}$thalassemia, patients have an increased risk of thromboembolic events, albeit lower than that in homozygous and $S / \beta^{0}$ patients. ${ }^{117,41}$ Considering that hemolytic and inflammatory processes are apparently less exacerbated in the majority of this subset of patients, ${ }^{118,119}$ compared to homozygous sickle cell anemia patients, the mechanisms behind this observation require further elucidation. In a retrospective analysis of 147 patients with these two conditions, higher hemoglobin levels and a history of surgical splenectomy were independently associated with the risk of VTE, ${ }^{120}$ suggesting that increased viscosity and RBC membrane alterations might be involved. ${ }^{121}$ However, it should also be noted that relatively increased levels of parameters indicative of hemolytic activity and inflammation have been associated with greater activation of coagulation and risk of VTE in patients with HbSC disease. ${ }^{122,123}$

The concept of thromboinflammation encompasses a broad spectrum of human disorders with both thrombotic and inflammatory manifestations, and it has been proposed that the level of thrombin would be a key determinant of the relative contribution of inflammation or thrombosis to disease presentation. ${ }^{50}$ According to this model, thrombotic microangiopathies and antiphospholipid syndrome are located in that part of the spectrum in which thrombotic manifestations are predominant, while rheumatoid arthritis and atherosclerosis are located on the opposite side of the spectrum, dominated by inflammatory mechanisms. Interestingly, SCD has been proposed to be located in the middle part of this postulated spectrum, ${ }^{50}$ reflecting the difficulty in characterizing the prominence of hemostasis acti- 
vation or inflammation in its pathogenesis. Additionally, the fact that infections represent a trigger for some SCD complications makes this characterization even more challenging. ${ }^{1}$ Accordingly, a recent review about the prothrombotic manifestations of SCD suggested that future clinical studies targeting coagulation activation should focus on a broader range of endpoints including end-organ damage. ${ }^{19} \mathrm{We}$ believe that this suggestion could be further expanded in the future by attempting to classify SCD complications as parts of a similar thromboinflammatory spectrum, in which the contributions of inflammation and hemostatic activation vary (Figure 2). Refinement of this model based on previous and future studies could allow a more precise definition of biomarkers and targets for the treatment of SCD.

\section{Perspectives}

Thromboinflammatory pathways, involving the activation of hemostasis and platelets, and prompted by RBC

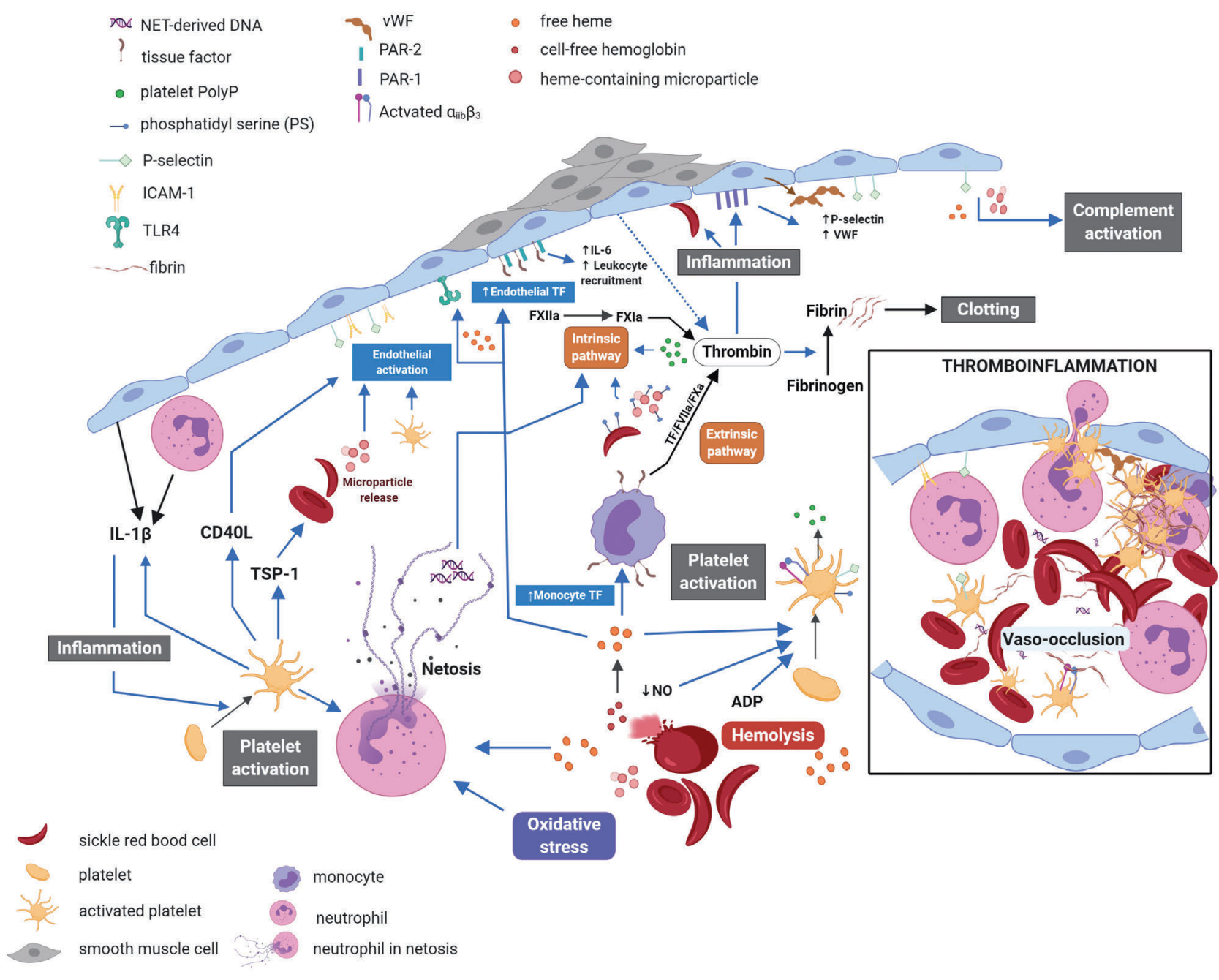

Figure 1. Thromboinflammatory pathways in sickle cell disease. Hemolytic and ischemia-reperfusion events (due to vaso-occlusion) drive inflammation and hemostatic alterations in sickle cell disease (SCD). Hemolysis leads to the release of cell-free hemoglobin, heme and other red blood cell contents into the vascular environment, causing nitric oxide depletion and contributing to platelet activation. Non-protein bound heme activates the endothelium (with some participation of tolllike receptor 4), induces tissue factor (TF) expression and activation in monocytes and endothelial cells, neutrophil extracellular trap formation, leukocyte and complement activation. Endothelial activation by platelets, leukocytes, ischemia-reperfusion events and heme/heme-laden microparticles results in the expression of adhesion molecules, including P-selectin and integrin $\alpha_{\mathrm{ib}} \beta_{3}$, which recruit leukocytes and, in turn, red cells to the blood vessel walls. Activated endothelial cells, leukocytes and platelets also produce mature interleukin-1 $\beta$, which further activates platelets and clot formation, in addition to having major inflammatory effects. Plateletderived proteins, such as $\mathrm{CD} 40 \mathrm{~L}$ and thrombospondin-1, activate the endothelium and may trigger red cell microparticle release. TF expression by monocytes leads to thrombin generation via the extrinsic (TF/FVIla/FXa) pathway, while TF expression in activated endothelium signals through PAR-2 receptors (TF/FVIla/FXa) triggering pro-inflammatory responses (interleukin-6 expression and leukocyte recruitment). Thrombin also mediates pro-inflammatory pathways through PAR-1 receptors, triggering von Willebrand factor release and P-selectin expression, which contribute to platelet adhesive mechanisms. Thrombin generation is further increased by phosphatidylserine-expressing red blood cells and microparticles, platelet polyPs and cell-free DNA derived from NET, via the intrinsic pathway of coagulation. Thrombin generation ultimately results in fibrin production and clotting, but also induces red cell adhesion to the vascular wall, in addition to inflammation through PAR-1 signaling. Oxidative stress contributes to NET formation and endothelial activation. These cellular responses are part of thromboinflammation, illustrated in the inset of figure 1. Leukocyte, platelet and red blood cell recruitment to the vascular wall, together with clotting processes, neutrophil extracellular trap components and the formation of heterocellular aggregates between platelets, leukocytes and red blood cells, with subsequent red cell trapping, results in the vaso-occlusive processes that characterize SCD. ADP: adenosine diphosphate; ICAM: intercellular adhesion molecule; IL: interleukin; NET: neutrophil extracellular trap; NO: nitric oxide; PAR: protease-activated receptor; TF: tissue factor; TLR4: toll-like receptor 4; TSP: thrombospondin; VWF: von Willebrand factor. Figure created with BioRender.com. 
alterations and destruction, and ensuing innate immune responses, play significant roles in some of the major complications of SCD, such as stroke, and exacerbate the chronic inflammation and cellular interactions that trigger vaso-occlusion, ischemia/reperfusion processes and eventually organ damage. As such, components of hemostasis and markers of platelet activation constitute therapeutic targets in SCD. Indeed, crizanlizumab, which was recently approved by the Food and Drug Administration for use in SCD, following clinical data from the phase II SUSTAIN trial ${ }^{124}$ that indicated that it may be effective for preventing painful vaso-occlusive crises, is an anti P-selectin therapy, which may benefit patients not just by diminishing endothelial receptivity to leukocyte adhesion, but by reducing the participation of platelets in the formation of heterocellular aggregates.

The most frequently employed therapy for SCD is hydroxyurea; while hydroxyurea provides clinical benefits by increasing fetal hemoglobin production, and therefore partially inhibiting $\mathrm{HbS}$ polymerization, this drug is an important anti-inflammatory agent. ${ }^{2}$ In addition to reducing the incidence of hospitalization and acute pain in SCD, hydroxyurea reduces the occurrence of acute chest syndrome and may provide an alternative to chronic transfusion therapy in some subjects at risk of stroke, ${ }^{125,126}$ indicating benefits on the hemostatic and thromboinflammatory mechanisms that contribute to these complica- tions. However, curiously, while an association of hydroxyurea therapy with reduced levels of hypercoagulability biomarkers has been reported in SCD, ${ }^{127}$ together with reductions in platelet-fibrinogen binding, leukocyte TF expression and complement activation, ${ }^{38,105,128}$ the effects of hydroxyurea on platelet-leukocyte aggregate formation ${ }^{129}$ and on the circulating concentrations of some platelet-derived pro-inflammatory molecules are less clear. $^{10,108} 128$

Anti-platelet therapies, while producing promising results in pre-clinical studies have not, thus far, been successful in larger clinical trials; prasugrel hydrochloride, an inhibitor of platelet activation, was apparently well-tolerated and safe in children and adolescents with SCD, but failed to reduce vaso-occlusive complications significantly when administered for up to 24 months. ${ }^{130}$ While concerns regarding the use of anticoagulants in SCD patients have been raised, the long-term administration of rivaroxaban in SCD patients with VTE was not associated with major bleeding events, ${ }^{131}$ although the use of vitamin $\mathrm{K}$ antagonists and low-molecular-weight heparin in such patients has been associated with some bleeding events. ${ }^{132}$ Thus, given the aforementioned range of complications that may be triggered by thrombosis and inflammation (see Figure 1), it may be important to tailor prospective therapies to target thromboinflammatory mechanisms that could be more specific to certain complications of SCD, such as

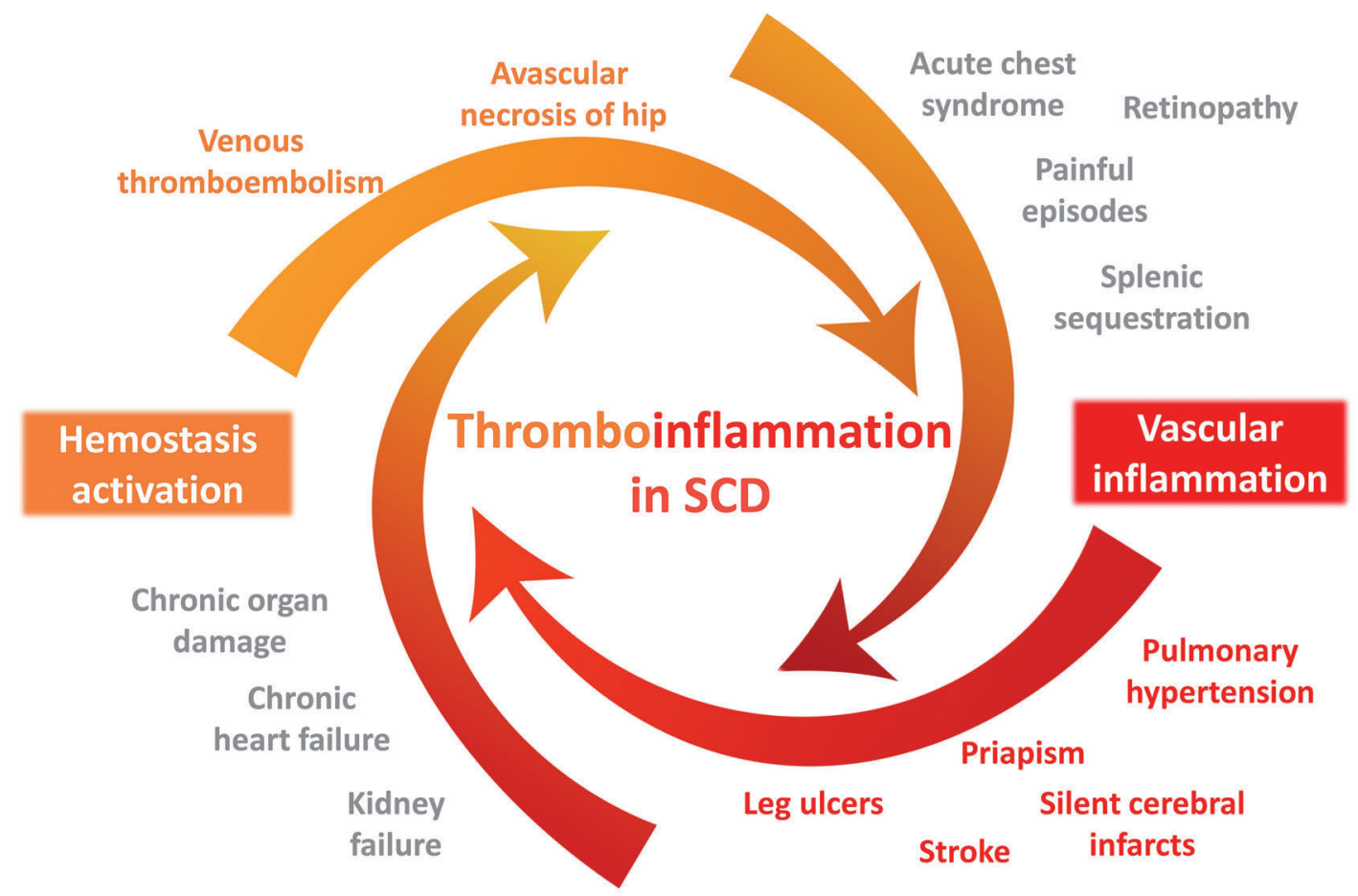

Figure 2. Complications of sickle cell disease and thromboinflammation. Thromboinflammation, or immunothrombosis, can be viewed as a physiological response to pathogens or tissue damage in which hemostasis activation and localized activation of inflammatory pathways at the microvascular level (i.e. vascular inflammation) act in concert to facilitate pathogen removal or tissue repair. However, loss of localization or deregulated activation of these pathways underlies the pathogenesis of a multitude of immune-mediated diseases in which the relative contribution of hemostasis and/or vascular inflammation varies, determining disease presentation, as proposed by Jackson et al..$^{50}$ Sickle cell disease (SCD) is a condition in which thromboinflammatory mechanisms have long been recognized as critical to its pathogenesis. However, the complex interplay between hemostasis and innate immunity activation makes it difficult to define precisely the relative contribution of each of these two processes to the pathogenesis of different complications, possibly explaining the absence of a straightforward association between classical biomarkers of hemostasis activation and the risk or the severity of some of these clinical manifestations. Accordingly, although it is likely that venous thromboembolism and avascular necrosis of the hip are complications in which hemostasis activation plays a dominant role (in orange), while pulmonary hypertension, priapism and leg ulcers can be more adequately placed in the vascular inflammation (in red) side of this thromboinflammatory spectrum, for most other complications, there is yet not enough evidence to precisely identify their localization in this cycle/spectrum, which could facilitate the identification of therapeutic targets, as well as biomarkers for each SCD complication. 
anticoagulants to avoid VTE, antiplatelet drugs for patients at risk of acute chest syndrome and anti-adhesive therapies to reduce vaso-occlusion and end-organ damage. Finally, given that thromboinflammation may be a major element in the pathogenesis of COVID-19, possibly contributing to both increased risk of VTE and to thrombosis in the lung microcirculation, pre-existing SCD thromboinflammation could represent a serious risk for complications from COVID-19 infection in this patient population. ${ }^{133}$

\section{Funding}

The authors of this review receive research funding from FAPESP (Fundação de Amparo à Pesquisa do Estado de S. Paulo), grant number: 2014/00984-3.

\section{References}

1. Kato GJ, Piel FB, Reid CD, et al. Sickle cell disease. Nat Rev Dis Primers. 2018;4:18010.

2. Steinberg $M H$. Overview of sickle cell anemia pathophysiology. In: Costa FF, Conran $N$, eds. Sickle Cell Anemia: From Basic Science To Clinical Practice. Switzerland: Springer International, 2016:49-75.

3. Wandersee NJ, Hillery CA. Red blood cells and the vaso-occlusive process. In: Costa FF, Conran N, eds. Sickle Cell Anemia: From Basic Science To Clinical Practice. New York: Springer Int., 2016:49-74.

4. Reiter CD, Wang X, Tanus-Santos JE, et al. Cell-free hemoglobin limits nitric oxide bioavailability in sickle-cell disease. Nat Med. 2002;8(12):1383-1389.

5. Schaer DJ, Buehler PW. Cell-free hemoglobin and its scavenger proteins: new disease models leading the way to targeted therapies. Cold Spring Harb Perspect Med. 2013;3(6):a013433.

6. Hebbel RP, Belcher JD, Vercellotti GM. The multifaceted role of ischemia/reperfusion in sickle cell anemia. J Clin Invest. 2020;130 (3):1062-1072.

7. Turhan A, Weiss LA, Mohandas N, Coller BS, Frenette PS. Primary role for adherent leukocytes in sickle cell vascular occlusion: a new paradigm. Proc Natl Acad Sci U S A. 2002;99(5):3047-3051.

8. Bennewitz MF, Jimenez MA, Vats $R$, et al. Lung vaso-occlusion in sickle cell disease mediated by arteriolar neutrophil-platelet microemboli. JCI Insight. 2017;2(1):e89761.

9. Conran N, Belcher JD. Inflammation in sickle cell disease. Clin Hemorheol Microcirc. 2018;68(2-3):263-299.

10. Dominical VM, Samsel L, Nichols JS, et al. Prominent role of platelets in the formation of circulating neutrophil-red cell heterocellular aggregates in sickle cell anemia. Haematologica. 2014;99(11):e214-217.

11. Proenca-Ferreira R, Brugnerotto AF, Garrido VT, et al. Endothelial activation by platelets from sickle cell anemia patients. PLoS One. 2014;9(2):e89012.

12. Nickel RS, Hsu LL. Clinical manifestations of sickle cell anemia: infants and children. In: Costa FF, Conran N, eds. Sickle Cell Anemia: From Basic Science To Clinical Practice. Switzerland: Springer International, 2016:213-29.

13. Zhang D, Chen G, Manwani D, et al. Neutrophil ageing is regulated by the microbiome. Nature. 2015;525(7570):528-532.

14. Dutta D, Methe B, Amar S, Morris A, Lim $\mathrm{SH}$. Intestinal injury and gut permeability in sickle cell disease. J Transl Med. 2019;17(1): 183.

15. Allali S, Maciel TT, Hermine O, de Montalembert M. Innate immune cells, major protagonists of sickle cell disease pathophysiology. Haematologica. 2020;105 (2):273-283.

16. Ataga KI, Key NS. Hypercoagulability in sickle cell disease: new approaches to an old problem. Hematology Am Soc Hematol Educ Program. 2007:91-96.

17. Faes C, Sparkenbaugh EM, Pawlinski R. Hypercoagulable state in sickle cell disease. Clin Hemorheol Microcirc. 2018;68(23):301-318

18. Nasimuzzaman M, Malik P. Role of the coagulation system in the pathogenesis of sickle cell disease. Blood Adv. 2019;3(20): 3170-3180.

19. Sparkenbaugh E, Pawlinski R. Prothrombotic aspects of sickle cell disease. J Thromb Haemost. 2017;15(7):1307-1316.

20. Nsiri B, Gritli N, Bayoudh F, Messaoud T, Fattoum S, Machghoul S. Abnormalities of coagulation and fibrinolysis in homozygous sickle cell disease. Hematol Cell Ther. 1996;38(3):279-284

21. Setty BN, Rao AK, Stuart MJ. Thrombophilia in sickle cell disease: the red cell connection. Blood. 2001;98(12):32283233 .

22. van Beers EJ, Schaap MC, Berckmans RJ, et al. Circulating erythrocyte-derived microparticles are associated with coagulation activation in sickle cell disease. Haematologica. 2009;94(11):1513-1519.

23. Lim MY, Ataga KI, Key NS. Hemostatic abnormalities in sickle cell disease. Curr Opin Hematol. 2013;20(5):472-477.

24. Key NS, Slungaard A, Dandelet L, et al. Whole blood tissue factor procoagulant activity is elevated in patients with sickle cell disease. Blood. 1998:91(11):4216-4223.

25. Setty BN, Key NS, Rao AK, et al. Tissue factor-positive monocytes in children with sickle cell disease: correlation with biomarkers of haemolysis. $\mathrm{Br} \mathrm{J}$ Haematol. 2012;157(3):370-380.

26. Solovey A, Gui L, Key NS, Hebbel RP. Tissue factor expression by endothelial cells in sickle cell anemia. J Clin Invest. 1998;101(9): 1899-1904.

27. Sins JWR, Schimmel M, Luken BM, et al. Dynamics of von Willebrand factor reactivity in sickle cell disease during vaso-occlusive crisis and steady state. J Thromb Haemost. 2017;15(7):1392-1402.

28. Shet AS, Aras O, Gupta K, et al. Sickle blood contains tissue factor-positive microparticles derived from endothelial cells and monocytes. Blood. 2003;102(7):2678-2683.

29. Schimmel M, Nur E, Biemond BJ, et al. Nucleosomes and neutrophil activation in sickle cell disease painful crisis. Haematologica. 2013;98(11):1797-1803.

30. Solovey A, Kollander R, Shet A, et al. Endothelial cell expression of tissue factor in sickle mice is augmented by hypoxia/reoxygenation and inhibited by lovastatin. Blood. 2004;104(3):840-846.

31. Chantrathammachart $\mathrm{P}$, Mackman $\mathrm{N}$, Sparkenbaugh E, et al. Tissue factor promotes activation of coagulation and inflammation in a mouse model of sickle cell disease. Blood. 2012;120(3):636-646.

32. Polanowska-Grabowska R, Wallace K, Field
JJ, et al. P-selectin-mediated platelet-neutrophil aggregate formation activates neutrophils in mouse and human sickle cell disease. Arterioscler Thromb Vasc Biol. 2010;30(12):2392-2399.

33. Sparkenbaugh EM, Chantrathammachart $\mathrm{P}$, Wang $S$, et al. Excess of heme induces tissue factor-dependent activation of coagulation in mice. Haematologica. 2015;100(3):308314.

34. Arumugam PI, Mullins ES, Shanmukhappa SK, et al. Genetic diminution of circulating prothrombin ameliorates multiorgan pathologies in sickle cell disease mice. Blood. 2015;126(15):1844-1855.

35. Sparkenbaugh EM, Chantrathammachart $\mathrm{P}$, Mickelson J, et al. Differential contribution of FXa and thrombin to vascular inflammation in a mouse model of sickle cell disease. Blood. 2014;123(11):1747-1756.

36. Nasimuzzaman M, Arumugam PI, Mullins $\mathrm{ES}$, et al. Elimination of the fibrinogen integrin alphaMbeta2-binding motif improves renal pathology in mice with sickle cell anemia. Blood Adv. 2019;3(9):1519-1532.

37. Faes C, Ilich A, Sotiaux A, et al. Red blood cells modulate structure and dynamics of venous clot formation in sickle cell disease. Blood. 2019;133(23):2529-2541.

38. Roumenina LT, Chadebech P, Bodivit G, et al. Complement activation in sickle cell disease: dependence on cell density, hemolysis and modulation by hydroxyurea therapy. Am J Hematol. 2020;95(5):456-464.

39. Vercellotti GM, Dalmasso AP, Schaid TR, Jr. et al. Critical role of $\mathrm{C} 5 \mathrm{a}$ in sickle cell disease. Am J Hematol. 2019;94(3):327-337.

40. Ohene-Frempong K, Weiner SJ, Sleeper LA et al. Cerebrovascular accidents in sickle cell disease: rates and risk factors. Blood. 1998;91(1):288-294.

41. Naik RP, Streiff MB, Haywood C Jr, Segal JB, Lanzkron S. Venous thromboembolism incidence in the Cooperative Study of Sickle Cell Disease. J Thromb Haemost. 2014;12 (12):2010-2016.

42. Noubouossie D, Key NS, Ataga KI. Coagulation abnormalities of sickle cell disease: relationship with clinical outcomes and the effect of disease modifying therapies. Blood Rev. 2016;30(4):245-256.

43. Sparkenbaugh E, Pawlinski R. Interplay between coagulation and vascular inflammation in sickle cell disease. Br J Haematol. 2013;162(1):3-14.

44. Ataga KI, Moore CG, Hillery CA, et al. Coagulation activation and inflammation in sickle cell disease-associated pulmonary hypertension. Haematologica. 2008;93(1): 20-26.

45. Ataga KI, Brittain JE, Desai P, et al. Association of coagulation activation with clinical complications in sickle cell disease. PLoS One. 2012;7(1):e29786.

46. Green D, Scott JP. Is sickle cell crisis a thrombotic event? Am J Hematol. 1986;23(4):317321.

47. van Beers EJ, Spronk HM, Ten Cate H, et al 

ischemia/reperfusion injury. Front Immunol. 2019;10:1260.

100. Mackman N. The role of the tissue factorthrombin pathway in cardiac ischemiareperfusion injury. Semin Vasc Med. 2003;3(2):193-198.

101. Senchenkova EY, Ansari J, Becker F, et al. Novel role for the AnxA1-Fpr2/ALX signaling axis as a key regulator of platelet function to promote resolution of inflammation. Circulation. 2019;140(4):319-335.

102. Rayes J, Bourne JH, Brill A, Watson SP. The dual role of platelet-innate immune cell interactions in thrombo-inflammation. Res Pract Thromb Haemost. 2020;4(1):23-35.

103. Vallance TM, Zeuner MT, Williams HF Widera D, Vaiyapuri S. Toll-like receptor 4 signalling and its impact on platelet function, thrombosis, and haemostasis. Mediators Inflamm. 2017;2017:9605894.

104. Liu FF, Tu TT, Zhang HF, et al. Coexpression network analysis of platelet genes in sickle cell disease. Platelets. 2019;30(8):1022-1029.

105. Proenca-Ferreira R, Franco-Penteado CF, Traina F, Saad ST, Costa FF, Conran N. Increased adhesive properties of platelets in sickle cell disease: roles for alphallb beta3mediated ligand binding, diminished cAMP signalling and increased phosphodiesterase 3 A activity. Br J Haematol. 2010;149(2):280288.

106.Lee SP, Ataga KI, Orringer EP, Phillips DR, Parise LV. Biologically active CD40 ligand is elevated in sickle cell anemia: potential role for platelet-mediated inflammation. Arterioscler Thromb Vasc Biol. 2006;26(7): 1626-1631.

107. Wun T, Paglieroni T, Rangaswami A, et al. Platelet activation in patients with sickle cell disease. Br J Haematol. 1998;100(4):741-749.

108. Novelli EM, Kato GJ, Ragni MV, et al. Plasma thrombospondin-1 is increased during acute sickle cell vaso-occlusive events and associated with acute chest syndrome, hydroxyurea therapy, and lower hemolytic rates. Am J Hematol. 2012;87(3):326-330.

109. Mehta P, Mehta J. Abnormalities of platelet aggregation in sickle cell disease. J Pediatr. 1980;96(2):209-213.

110. Villagra J, Shiva S, Hunter LA, Machado RF, Gladwin MT, Kato GJ. Platelet activation in patients with sickle disease, hemolysis-associated pulmonary hypertension, and nitric oxide scavenging by cell-free hemoglobin. Blood. 2007;110(6):2166-2172.

111.Li J, Kim K, Jeong SY, et al. Platelet protein disulfide isomerase promotes glycoprotein Ibalpha-mediated platelet-neutrophil inter- actions under thromboinflammatory conditions. Circulation. 2019;139(10):1300-1319.

112. Kim K, Li J, Barazia A, et al. ARQ 092, an orally-available, selective AKT inhibitor, attenuates neutrophil-platelet interactions in sickle cell disease. Haematologica. 2017;102 (2):246-259.

113. Garrido VT, Proenca-Ferreira R, Dominical $\mathrm{VM}$, et al. Elevated plasma levels and platelet-associated expression of the prothrombotic and pro-inflammatory protein, TNFSF14 (LIGHT), in sickle cell disease. Br J Haematol. 2012;158(6):788-797.

114. Camus SM, Gausseres B, Bonnin P, et al. Erythrocyte microparticles can induce kidney vaso-occlusions in a murine model of sickle cell disease. Blood. 2012;120(25):50505058.

115. Vogel S, Arora T, Wang X, et al. The platelet NLRP3 inflammasome is upregulated in sickle cell disease via HMGB1/TLR4 and Bruton tyrosine kinase. Blood Adv. 2018;2(20):2672-2680.

116. Sparkenbaugh EM, Chantrathammachart $P$ Chandarajoti K, Mackman N, Key NS, Pawlinski R. Thrombin-independent contribution of tissue factor to inflammation and cardiac hypertrophy in a mouse model of sickle cell disease. Blood. 2016;127(10):13711373.

117. Pecker LH, Schaefer BA, Luchtman-Jones L. Knowledge insufficient: the management of haemoglobin SC disease. Br J Haematol. 2017;176(4):515-526.

118. da Guarda CC, Yahouedehou S, Santiago RP, et al. Sickle cell disease: a distinction of two most frequent genotypes (HbSS and $\mathrm{HbSC}$ ). PLoS One. 2020;15(1):e0228399.

119. Nagel RL, Fabry ME, Steinberg MH. The paradox of hemoglobin SC disease. Blood Rev. 2003;17(3):167-178.

120. Yu TT, Nelson J, Streiff MB, Lanzkron S, Naik RP. Risk factors for venous thromboembolism in adults with hemoglobin SC or Sbeta $(+)$ thalassemia genotypes. Thromb Res. 2016;141:35-38.

121.Lionnet F, Hammoudi N, Stojanovic KS, et al. Hemoglobin sickle cell disease complications: a clinical study of 179 cases. Haematologica. 2012;97(8):1136-1141.

122. Colella MP, de Paula EV, Machado-Neto JA, et al. Elevated hypercoagulability markers in hemoglobin SC disease. Haematologica. 2015;100(4):466-471.

123. Saad STO, Gilli SO. Hemoglobin Sbeta thalassemia, SC disease, and SD disease. In: Costa FF CN, ed. Sickle Cell Anemia: From Basic Science To Clinical Practice.
Switzerland: Springer International, 2016: 319-338.

124. Ataga KI, Kutlar A, Kanter J, et al Crizanlizumab for the prevention of pain crises in sickle cell disease. $N$ Engl J Med. 2017;376(5):429-439.

125. Charache S, Barton FB, Moore RD, et al Hydroxyurea and sickle cell anemia. Clinical utility of a myelosuppressive "switching" agent. The Multicenter Study of Hydroxyurea in Sickle Cell Anemia. Medicine (Baltimore). 1996;75(6):300-326.

126. Ware RE, Davis BR, Schultz WH, et al. Hydroxycarbamide versus chronic transfusion for maintenance of transcranial doppler flow velocities in children with sickle cell anaemia-TCD With Transfusions Changing to Hydroxyurea (TWiTCH): a multicentre, open-label, phase 3, non-inferiority trial. Lancet. 2016;387(10019):661-670.

127. Colella MP, De Paula EV, Conran N, et al Hydroxyurea is associated with reductions in hypercoagulability markers in sickle cell anemia. J Thromb Haemost. 2012;10(9): 1967-1970.

128. Gumiero D, Di Gennaro L, Nicolazzi MA, Landolfi R. Hydroxyurea-mediated release of nitric oxide in myeloproliferative neoplasms patients: effects on platelet-leukocyte interaction. J Clin Pharmacol 2015;55(10):1125-1130

129. Trelinski J, Tybura M, Smolewski P, Robak T, Chojnowski K. The influence of low-dose aspirin and hydroxyurea on platelet-leukocyte interactions in patients with essential thrombocythemia. Blood Coagul Fibrinolysis. 2009;20(8):646-651.

130. Heeney MM, Hoppe CC, Abboud MR, et al. A multinational trial of prasugrel for sickle cell vaso-occlusive events. N Engl J Med. 2016;374(7):625-635.

131. Christen JR, Bertolino J, Jean E, et al. Use of direct oral anticoagulants in patients with sickle cell disease and venous thromboembolism: a prospective cohort study of 12 patients. Hemoglobin. 2019:43(4-5):296299.

132. Patel A, Williams H, Baer MR, Zimrin AB, Law JY. Decreased bleeding incidence with direct oral anticoagulants compared to vitamin K antagonist and low-molecular-weight heparin in patients with sickle cell disease and venous thromboembolism. Acta Haematol. 2019;142(4):233-238.

133. Connors JM, Levy JH. Thromboinflammation and the hypercoagulability of COVID-19. J Thromb Haemost. 2020 Apr 17. [Epub ahead of print] 